\title{
NICE HOMOLOGY COALGEBRAS
}

\author{
BY \\ A. K. BOUSFIELD( ${ }^{(1)}$
}

1. Introduction. Several different unstable versions of the Adams spectral sequence for homotopy groups have recently been constructed ([3], [9], [14]). While these spectral sequences differ for general spaces, it will be shown in [2] that they coincide (at least mod 2) for spaces having nice (2.2) homology coalgrebras, e.g. for spheres and loop spaces but not for $S^{2} \vee S^{2}$. The purpose of this note is to prove two purely algebraic results on nice homology coalgebras which will be needed in [2]. The first (4.1) provides a homological characterization of niceness, and the second $(\S 6)$ gives the structure of the Hopf algebra $\operatorname{Cotor}^{C}(k, k)$ when $C$ is a nice $k$-coalgebra. Throughout this note we employ a theory of derived functors for nonadditive functors which is due to André [1], Quillen [13], and others. For convenience, we outline a simplified version of this theory in an appendix ( $\$ 7)$.

The definition (2.2) of niceness for a homology coalgebra $C$ comes essentially from Moore-Smith [12,\$4] and involves the existence of a certain presentation for $C$. Our homological characterization of niceness is based on the notion of primitive dimension (3.1). In particular we study (\$3) right derived functors $R^{n} P$ of the primitive element functor $P$, and we show (4.1) that a homology coalgebra $C$ is nice if and only if $R^{n} P(C)=0$ for $n>1$, i.e. $C$ has primitive dimension $\leqq 1$.

For a homology coalgebra $C$ over a field $k, R^{*} P(C)$ is closely related to the Hopf algebra $\operatorname{Cotor}^{C}(k, k)$ defined by Eilenberg-Moore [7] (see (5.1)). We construct (5.3) a spectral sequence of Hopf algebras whose $E^{2}$-term depends only on $R^{*} P(C)$ and which converges to $\operatorname{Cotor}^{C}(k, k)$. Using this we determine (\$6) the structure of $\operatorname{Cotor}^{C}(k, k)$ when $C$ is nice, and we thereby generalize certain results of [12]. It should be noted that when $C$ is of finite type $\operatorname{Cotor}^{C}(k, k)$ is isomorphic to $\operatorname{Ext}_{C^{*}}(k, k)$ and thus $\S 6$ gives information on the cohomology of algebras.

Finally the author wishes to acknowledge that results similar to 4.1 and 6.1 for commutative rings have been proved by D. G. Quillen [16] using different methods.

2. Homology coalgebras and their derived functors. Let $k$ be a fixed field. A homology $k$-coalgebra is a connected positively graded $k$-coalgebra with commutative comultiplication [11]. We let $\mathscr{C} / k$ denote the category of homology $k$-coalgebras. Thus $H_{*}(X ; k) \in \mathscr{C} \mid k$ for any connected space $X$.

Received by the editors February 12, 1969.

( $\left.{ }^{1}\right)$ Research supported in part by NSF GP 8885 .

Copyright (C) 1970, American Mathematical Society 
If $V$ is a strictly positively graded $k$-module, let $U(V)$ be the homology $k$ coalgebra cogenerated by $V$ (see [12]). This coalgebra is characterized by the existence of a map of graded $k$-modules

$$
\pi(V): U(V) \rightarrow V
$$

satisfying the universal mapping property: if $C \in \mathscr{C} \mid k$ and $f: C \rightarrow V$ is a map of graded $k$-modules, then there is a unique map $\bar{f}: C \rightarrow U(V)$ in $\mathscr{C} \mid k$ such that $\pi(V) \circ \bar{f}=f$. A coalgebra $C \in \mathscr{C} \mid k$ is cofree if $C \approx U(V)$ for some $V$.

DEFINITION 2.1. A sequence

$$
k \longrightarrow C^{\prime} \stackrel{f^{\prime}}{\longrightarrow} C \stackrel{f^{\prime \prime}}{\longrightarrow} C^{\prime \prime} \longrightarrow k
$$

in $\mathscr{C} \mid k$ is an injective extension sequence [12] if:

(i) $f^{\prime \prime}$ is an epimorphism (i.e. $f^{\prime \prime}$ is a surjection of the underlying graded $k$ modules).

(ii) $C$ is injective as a $C^{\prime \prime}$ comodule.

(iii) $f^{\prime}$ is the kernel of $f^{\prime \prime}$ (i.e. $f^{\prime}$ is the natural map $C \square_{C^{\prime \prime}} k \rightarrow C$ of [11]).

Definition 2.2. A coalgebra $C^{\prime} \in \mathscr{C} \mid k$ is nice if there exists an injective extension sequence

$$
k \longrightarrow C^{\prime} \stackrel{f^{\prime}}{\longrightarrow} C \stackrel{f^{\prime \prime}}{\longrightarrow} C^{\prime \prime} \longrightarrow k
$$

such that $C$ and $C^{\prime \prime}$ are cofree.

2.3. EXAMPLES. A coalgebra $B \in \mathscr{C} \mid k$ is nice under any of the following conditions:

(i) $B$ is the coalgebra of some Hopf algebra (see [12, §4]).

(ii) $B$ is of finite type and its dual $B^{*}$ is an algebra on one generator (or more generally $B^{*}$ is the quotient of a free commutative algebra by a Borel ideal [15, p. 79]).

(iii) $B \approx B^{\prime} \otimes_{k} B^{\prime \prime}$ where $B^{\prime}, B^{\prime \prime} \in \mathscr{C} \mid k$ are nice.

2.4. Derived functors of coalgebras. We now indicate how the general theory of derived functors in the Appendix ( $(7)$ applies to homology coalgebras. The class $\mathscr{M}$ of all cofree coalgebras in $\mathscr{C} \mid k$ is a class of injective models (7.2) for $\mathscr{C} \mid k$ by 7.8 . Thus if $T: \mathscr{C} \mid k \rightarrow \mathscr{A}$ is a (covariant) functor to an abelian category $\mathscr{A}$, then $T$ has right derived functors (with respect to $\mathscr{M}$ )

$$
\begin{gathered}
R^{n} T: \mathscr{C} \mid k \rightarrow \mathscr{A}, \quad n \geqq 0, \\
\varepsilon: T \rightarrow R^{0} T .
\end{gathered}
$$

To construct these explicitly, let $\mathscr{V} / k$ be the category of strictly positively graded $k$-modules and let

$$
J: \mathscr{C}|k \rightarrow \mathscr{V}| k, \quad U: \mathscr{V}|k \rightarrow \mathscr{C}| k
$$

be the adjoint functors with $U$ as above and with $J(C) \in \mathscr{V} \mid k$ equal to $C$ in all positive degrees. Letting $K=U J$, there is given by 7.7 an augmented cosimplicial (7.6) object $K C$ for each $C \in \mathscr{C} \mid k$, with $K C^{n}=K^{n+1} C$ for $n \geqq-1$. We call $K C$ the 
canonical resolution of $C$. Let $\tilde{K} C$ equal $K C$ without the augmentation. By 7.8 and 7.9

$$
R^{n} T(C) \approx H^{n}(T \tilde{K} C), \quad n \geqq 0,
$$

and $\varepsilon: T(C) \rightarrow R^{0} T(C)$ is induced by the augmentation of $K C$.

For our applications we need a more general type of resolution than $K C$.

Definition 2.5. A cosimplicial resolution of $C \in \mathscr{C} \mid k$ consists of an augmented cosimplicial object $\boldsymbol{X}$ over $\mathscr{C} \mid k$ such that:

(i) $\boldsymbol{X}^{-1}=C$.

(ii) For $n \geqq 0, X^{n}$ is cofree.

(iii) For $n \geqq-1, H^{n}(J \boldsymbol{X})=0$.

Let $\tilde{\boldsymbol{X}}$ denote $\boldsymbol{X}$ without the augmentation.

REMARK 2.6. It is easily shown that $K C$ is a cosimplicial resolution of $C$.

Proposition 2.7. If $\boldsymbol{X}$ is a cosimplicial resolution of $C \in \mathscr{C} \mid k$ and $T: \mathscr{C} \mid k \rightarrow \mathscr{A}$ is as above, then there are natural isomorphisms

$$
R^{n} T(C) \approx H^{n}(T \tilde{X}), \quad n \geqq 0 .
$$

Proof. We must show that the chain complex $\mathrm{ch}^{+} \boldsymbol{X}(7.6)$ is a right $\mathscr{M}$-resolution (7.1) of $C$. For the acyclicity condition 7.1(ii) it suffices, by the adjointness of $U$ and $J$, to show that the chain complex of the augmented simplicial abelian group

$$
Z \operatorname{Hom}_{\mathscr{V} / k}(J X, V), \quad V \in \mathscr{V} \mid k
$$

is acyclic, where $Z(\mathrm{)}$ is the free abelian group functor. This follows by standard simplicial methods (see [10]) since the chain complex of the augmented simplicial $k$-module $\operatorname{Hom}_{\mathscr{V} / k}(J X, V)$ is acyclic.

3. Primitive dimension of coalgebras. Let

$$
R^{n} P: \mathscr{C}|k \rightarrow \mathscr{V}| k, \quad n \geqq 0
$$

be the right derived functors (2.4) of the primitive [11] element functor $P$ for homology $k$-coalgebras.

Definition 3.1. A coalgebra $C \in \mathscr{C} \mid k$ has primitive dimension $\leqq d$ if $R^{n} P(C)=0$ for all $n>d$.

In this section we develop the general properties of the functors $R^{n} P$, and leave unstated the obvious corollaries on primitive dimension.

Proposition 3.2. For each $C \in \mathscr{C} \mid k, \varepsilon: P(C) \underset{\rightarrow}{\rightarrow} R^{0} P(C)$ is an isomorphism.

Proof. By the definition of $P$ there is an exact sequence

$$
0 \rightarrow P \rightarrow J \rightarrow J \otimes J
$$

and this induces an exact sequence

$$
0 \rightarrow R^{0} P \rightarrow R^{0} J \rightarrow R^{0}(J \otimes J)
$$


Clearly

$$
\varepsilon: J \stackrel{\approx}{\longrightarrow} R^{0} J, \quad \varepsilon: J \otimes J \stackrel{\approx}{\longrightarrow} R^{0}(J \otimes J)
$$

and so $\varepsilon: P \stackrel{\approx}{\rightarrow} R^{0} P$.

Proposition 3.3. If $B, C \in \mathscr{C} \mid k$ then

$$
R^{n} P(B \otimes C) \approx R^{n} P(B) \oplus R^{n} P(C), \quad n \geqq 0 .
$$

Proof. If $\boldsymbol{X}, \boldsymbol{Y}$ are cosimplicial resolutions (2.5) for $B, C$, then the degreewise tensor product $\boldsymbol{X} \otimes \boldsymbol{Y}$ is a cosimplicial resolution for $B \otimes C$ by the EilenbergZilber theorem (see [5]). Since

$$
P(\tilde{\boldsymbol{X}} \otimes \tilde{\boldsymbol{Y}}) \approx P \tilde{\boldsymbol{X}} \oplus P \tilde{\boldsymbol{Y}}
$$

the result follows.

Proposition 3.4. A map $h: B \rightarrow C$ in $\mathscr{C} \mid k$ is an isomorphism if and only if

$$
R^{n} P(h): R^{n} P(B) \rightarrow R^{n} P(C)
$$

is an isomorphism for $n=0$ and a monomorphism for $n=1$.

An equivalent result is given in [12,3.2]. For our proof we take $X$ and $\widetilde{K} B$ and $\boldsymbol{Y}=\tilde{\boldsymbol{K}} C$ in the following lemma.

Lemma 3.5. Let $\boldsymbol{f}: \boldsymbol{X} \rightarrow \boldsymbol{Y}$ be a map of dimensionwise cofree cosimplicial objects over $\mathscr{C} \mid k$. If

$$
(P \boldsymbol{f})^{*}: H^{q}(P X) \rightarrow H^{q}(P \boldsymbol{Y})
$$

is isomorphic for $q \leqq n$ and monomorphic for $q=n+1$, then (viewing $\boldsymbol{X}, \boldsymbol{Y}$ as cosimplicial graded $k$-modules)

$$
\boldsymbol{f}^{*}: H^{q}(\boldsymbol{X}) \rightarrow H^{q}(\boldsymbol{Y})
$$

is isomorphic for $q \leqq n$ and monomorphic for $q=n+1$.

Proof. Filter $\boldsymbol{X}$ by cosimplicial graded $k$-modules $\left\{F_{s} X\right\}_{s \geq 0}$ where $F_{s} X$ is the kernel of the composite

$$
X \stackrel{\Delta_{n+1}}{\longrightarrow} X^{(n+1)} \longrightarrow(J X)^{(n+1)}
$$

with $\Delta_{n+1}$ the $(n+1)$-fold diagonal. The resulting spectral sequence $\left\{E_{r} X\right\}_{r \geq 0}$ converges to $H^{*}(X)$, and there is a natural isomorphism $E_{0} X \approx U(P X)$ since each $X^{q}$ is cofree. The hypothesis of 3.5 implies [5] that

$$
(U P f)^{*}: H^{q}(U P X) \rightarrow H^{q}(U P Y)
$$

is isomorphic for $q \leqq n$ and monomorphic for $q=n+1$. A simple spectral sequence argument now completes the proof. 
THEOREM 3.6. Let

$$
k \longrightarrow C^{\prime} \stackrel{i}{\longrightarrow} C \stackrel{j}{\longrightarrow} C^{\prime \prime} \longrightarrow k
$$

be an injective extension sequence in $\mathscr{C} \mid k$. Then there is a natural long exact sequence

$$
\begin{gathered}
0 \longrightarrow R^{0} P\left(C^{\prime}\right) \stackrel{i_{*}}{\longrightarrow} R^{0} P(C) \longrightarrow \cdots \\
\longrightarrow R^{n-1} P\left(C^{\prime \prime}\right) \stackrel{\delta}{\longrightarrow} R^{n} P\left(C^{\prime}\right) \stackrel{i_{*}}{\longrightarrow} R^{n} P(C) \stackrel{j_{*}}{\longrightarrow} R^{n} P\left(C^{\prime \prime}\right) \stackrel{\delta}{\longrightarrow} \cdots
\end{gathered}
$$

with $i_{*}, j_{*}$ induced by $i, j$.

This extends the six term exact sequence of $[12,3.3]$. The proof will occupy the rest of $\S 3$.

3.7. Mapping cones. Let $\boldsymbol{f}: \boldsymbol{X} \rightarrow \boldsymbol{Y}$ be a map of cosimplicial objects over $\mathscr{C} \mid k$. The mapping cone $\boldsymbol{M}(\boldsymbol{f})$ is the cosimplicial object over $\mathscr{C} \mid k$ given by:

(i) $\boldsymbol{M}(\boldsymbol{f})^{0}=\boldsymbol{X}^{0}, \boldsymbol{M}(\boldsymbol{f})^{n}=\boldsymbol{X}^{n} \otimes \boldsymbol{Y}^{n-1} \otimes \cdots \otimes \boldsymbol{Y}^{0}, n \geqq 1$.

(ii) $d^{0}: \boldsymbol{M}(\boldsymbol{f})^{n} \rightarrow \boldsymbol{M}(\boldsymbol{f})^{n+1}$ equals $\left(\left(d^{0} \otimes \boldsymbol{f}\right) \Delta\right) \otimes 1 \otimes \cdots \otimes 1$ where $\Delta: \boldsymbol{X}^{n}$ $\rightarrow \boldsymbol{X}^{n} \otimes \boldsymbol{X}^{n}$ is the comultiplication.

(iii) $d^{i}: \boldsymbol{M}(\boldsymbol{f})^{n} \rightarrow \boldsymbol{M}(\boldsymbol{f})^{n+1}, \quad 0<i<n+1$ equals $d^{i} \otimes \cdots \otimes d^{1} \otimes\left(\left(d^{0} \otimes 1\right) \Delta\right)$ $\otimes 1 \otimes \cdots \otimes 1$ where $\Delta: \boldsymbol{Y}^{n-i} \rightarrow \boldsymbol{Y}^{n-i} \otimes \boldsymbol{Y}^{n-i}$ is the comultiplication.

(iv) $d^{n+1}: \boldsymbol{M}(\boldsymbol{f})^{n}=\boldsymbol{M}(\boldsymbol{f})^{n} \otimes k \rightarrow \boldsymbol{M}(\boldsymbol{f})^{n+1}$ equals $d^{n+1} \otimes \cdots \otimes d^{1} \otimes \eta$ where $\eta: k \rightarrow \boldsymbol{Y}^{0}$ is the coaugmentation.

(v) $s^{i}: \boldsymbol{M}(\boldsymbol{f})^{n+1} \rightarrow \boldsymbol{M}(\boldsymbol{f})^{n}, 0 \leqq i<n+1$ equals $s^{i} \otimes \cdots \otimes s^{0} \otimes \alpha \otimes 1 \otimes \cdots \otimes 1$ where $\alpha: Y^{n-i} \rightarrow k$ is the counit.

For any $B \in \mathscr{C} \mid k$ let $c(B)$ denote the constant cosimplicial object with $c(B)^{n}=B$ for all $n \geqq 0$.

Proposition 3.8. If $g: B^{\prime} \rightarrow B \in \mathscr{C} \mid k$ and $c g: c\left(B^{\prime}\right) \rightarrow c(B)$ is the induced cosimplicial map, then

$$
H^{n}(M(c g)) \approx \operatorname{Cotor}_{-n, *}^{B}\left(B^{\prime}, k\right), \quad n \geqq 0 .
$$

Proof. For $1: B \rightarrow B$, the cosimplicial object $\boldsymbol{M}(\boldsymbol{c} 1)$ augmented by $d^{0}: k \rightarrow M(c 1)^{0}$ may be viewed as an augmented cosimplicial object over the category of left $B$ comodules. The associated chain complex is an injective resolution of the left $B$ comodule $k$. (It is essentially the cobar resolution.) Since $M(c g) \approx B^{\prime} \square_{B} M(c 1)$, where $\square$ is the cotensor product [11], the result follows.

Let

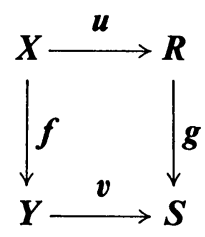

be a commutative diagram of cosimplicial objects over $\mathscr{C} \mid k$. 
Proposition 3.9. If

$$
\boldsymbol{u}^{*}: H^{*}(\boldsymbol{X}) \rightarrow H^{*}(\boldsymbol{R}), \quad \boldsymbol{v}^{*}: H^{*}(\boldsymbol{Y}) \rightarrow H^{*}(\boldsymbol{S})
$$

are isomorphic, then

$$
(\boldsymbol{u}, \boldsymbol{v})^{*}: H^{*}(\boldsymbol{M}(\boldsymbol{f})) \rightarrow H^{*}(\boldsymbol{M}(\boldsymbol{g}))
$$

is isomorphic.

Proof. First suppose $\boldsymbol{X}, \boldsymbol{Y}, \boldsymbol{R}, \boldsymbol{S}$ are dimensionwise cofree and that

$$
(P \boldsymbol{u})^{*}: H^{*}(P X) \rightarrow H^{*}(P \boldsymbol{R}), \quad(P \boldsymbol{v})^{*}: H^{*}(P \boldsymbol{Y}) \rightarrow H^{*}(P S)
$$

are isomorphisms. It is not hard to show

$$
H^{*}(P M f / P X) \approx H^{*-1}(P Y), \quad H^{*}(P M g / P R) \approx H^{*-1}(P S)
$$

and hence by the five lemma $\boldsymbol{u}, \boldsymbol{v}$ induce $H^{*}(P \boldsymbol{M f}) \approx H^{*}(\boldsymbol{P M g})$. Thus

$$
(\boldsymbol{u}, \boldsymbol{v})^{*}: H^{*}(\boldsymbol{M f}) \rightarrow H^{*}(\boldsymbol{M g})
$$

is isomorphic by 3.5 .

For the general case consider the diagram of cosimplicial objects

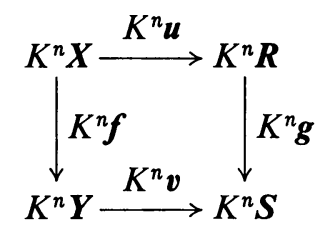

where $n \geqq 1$ and $K^{n}$ is as in 2.4. Since $P K^{n} X$ is of the form $T(J X)$ it follows by [5] that the diagram satisfies the hypotheses of our first case, and hence

$$
H^{*}\left(M K^{n} f\right) \stackrel{\approx}{\longrightarrow} H^{*}\left(M K^{n} g\right)
$$

is an isomorphism. There is a double cosimplicial object $\boldsymbol{M} \tilde{\boldsymbol{K}} \boldsymbol{f}$ (and also $\boldsymbol{M} \tilde{K} \boldsymbol{g}$ ) with

$$
(M \tilde{K} f)^{i, j}=\left(M K^{i+1} f\right)^{j}
$$

and we consider the two natural spectral sequences converging to the total homology $H^{*}(\boldsymbol{M} \tilde{\boldsymbol{K}} \boldsymbol{f})$, viewing $\boldsymbol{M} \tilde{\boldsymbol{K}} \boldsymbol{f}$ as a double chain complex. One spectral sequence shows

$$
H^{*}(\boldsymbol{M K} \tilde{f}) \rightarrow H^{*}(\boldsymbol{M K} \boldsymbol{g})
$$

is an isomorphism because of $\left(^{*}\right)$, and the other spectral sequence collapses to show

$$
H^{*}(\boldsymbol{M f}) \approx H^{*}(\boldsymbol{M} \tilde{\mathrm{K}}), \quad H^{*}(\boldsymbol{M g}) \approx H^{*}(\boldsymbol{M} \tilde{\mathbf{K} g})
$$

which proves the proposition.

3.10. Proof of 3.6. Let

$$
k \longrightarrow C^{\prime} \stackrel{i}{\longrightarrow} C \stackrel{j}{\longrightarrow} C^{\prime \prime} \longrightarrow k
$$


be an injective extension sequence. There is a commutative diagram

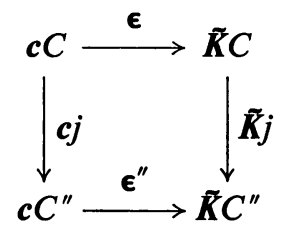

where $\epsilon, \epsilon^{\prime \prime}$ are induced by the augmentations of $K C, K C^{\prime \prime}$. Since

$$
\begin{aligned}
\operatorname{Cotor}_{-n, *}^{C^{\prime \prime}}(C, k) & =0, & & n>0, \\
& =C^{\prime}, & & n=0,
\end{aligned}
$$

3.8 and 3.9 imply that $\boldsymbol{M}(\tilde{K} j)$ is a cosimplicial resolution (2.5) of $C^{\prime}$ with augmentation omitted. Thus

$$
R^{n} P\left(C^{\prime}\right) \approx H^{n}(P M(\tilde{K} j)), \quad n \geqq 0 .
$$

The commutative diagram

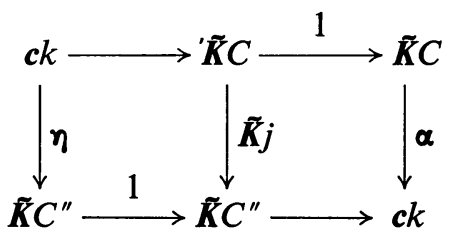

gives a sequence of mapping cones

$$
M(\eta) \rightarrow M(\tilde{K} j) \rightarrow M(\alpha)=\tilde{K} C .
$$

Applying $P$ we obtain a short exact sequence

$$
0 \rightarrow P M(\eta) \rightarrow P M(\tilde{K} j) \rightarrow P \tilde{K} C \rightarrow 0
$$

with

$H^{n}(P M(\eta)) \approx R^{n-1} P\left(C^{\prime \prime}\right), \quad H^{n}(P M(\tilde{K} j)) \approx R^{n} P\left(C^{\prime}\right), \quad H^{n}(P \tilde{K} C) \approx R^{n} P(C)$.

The first isomorphism is given by the coboundary map for the sequence of type $\left(^{*}\right)$ obtained using the injective extension sequence

$$
k \longrightarrow k \longrightarrow C^{\prime \prime} \stackrel{1}{\longrightarrow} C^{\prime \prime} \longrightarrow k .
$$

The cohomology sequence of $(*)$ is the exact sequence required for 3.6.

\section{Niceness and primitive dimension.}

THeOREM 4.1. A coalgebra $C \in \mathscr{C} \mid k$ is cofree if and only if $C$ is of primitive dimension $\leqq 0$, and $C$ is nice if and only if $C$ is of primitive dimension $\leqq 1$.

Proof. If $C$ is cofree then $R^{n} P(C)=0, n \geqq 1$, by 7.4. If $C$ is nice there is an injective extension sequence

$$
k \rightarrow C \rightarrow B \rightarrow B^{\prime \prime} \rightarrow k
$$


with $B$ and $B^{\prime \prime}$ cofree. The exact sequence 3.6 shows that $R^{n} P(C)=0, n \geqq 2$. The theorem will now follow from 4.2 and 4.4.

Proposition 4.2. If $R^{1} P(C)=0$ for $C \in \mathscr{C} \mid k$ then $C$ is cofree.

Proof. Choose a map $j: C \rightarrow P C$ of graded vector spaces with $j i=1$ where $i: P C \subset C$. Let $j: C \rightarrow U(P C)$ be the induced (\$2) map of coalgebras. Then 3.4 shows that $j$ is isomorphic, so $C$ is cofree.

Lemma 4.3. Let $j: B \rightarrow B^{\prime \prime}$ in $\mathscr{C} \mid k$ be such that

$$
\operatorname{Cotor}_{-1, *}^{B^{\prime \prime}}(B, k)=0 \text {. }
$$

Then

$$
k \longrightarrow B \square_{B^{\prime \prime}} k \stackrel{i}{\longrightarrow} B \stackrel{j}{\longrightarrow} B^{\prime \prime} \longrightarrow k
$$

is an injective extension sequence where $i$ is the natural inclusion.

Proof. Let $C$ denote $B \square_{B^{\prime \prime}} k$, and choose a map $r: B \rightarrow C$ of graded vector spaces such that $r i=1$. Let $h: B \rightarrow C \otimes B^{\prime \prime}$ be the extension [11, p. 221] of $r$ to a map of right $B^{\prime \prime}$ comodules. Then $h$ is monomorphic by [11, 2.5] since $h$ induces an isomorphism

$$
B \square_{B^{\prime \prime}} k \stackrel{\approx}{\longrightarrow}\left(C \otimes B^{\prime \prime}\right) \square_{B^{\prime \prime}} k .
$$

Also $h$ is epimorphic since its cokernel $D$ satisfies $D \square_{B^{\prime \prime}} k=0$ as shown by the long exact sequence for $\operatorname{Cotor}^{B^{\prime \prime}}(\cdot, k)$. Thus $h$ is an isomorphism of right $B^{\prime \prime}$ comodules and the lemma follows.

Proposition 4.4. If $R^{2} P(C)=0$ for $C \in \mathscr{C} / k$, then $C$ is nice.

Proof. Let $\boldsymbol{X}$ be any cosimplicial resolution (2.5) of $C$, and consider the normalized chain complex $N P X$ of $P X$ :

$$
\begin{gathered}
N P X^{0}=P X^{0}, \quad N P X^{n}=P X^{n} /\left(\operatorname{Im} d^{1}+\cdots+\operatorname{Im} d^{n}\right), \quad n \geqq 1, \\
\delta: N P X^{n-1} \rightarrow N P X^{n},
\end{gathered}
$$

with $\delta$ induced by the operator $d^{0}$.

By the normalization theorem (see [5]) $H^{2}(N P X) \approx H^{2}(P X) \approx R^{2} P(C)=0$.

Choose a map $h: N P X^{1} \rightarrow M \in \mathscr{V} / k$ such that the chain map

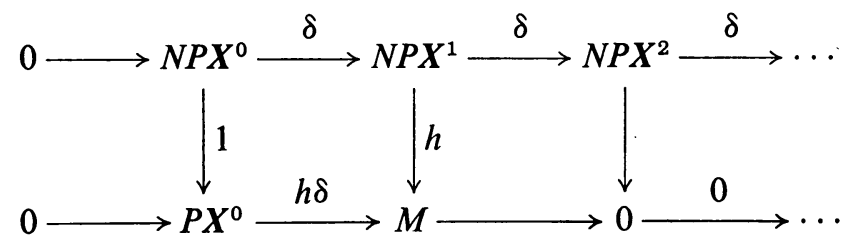

induces cohomology isomorphisms in degrees $\leqq 2$.

Now construct a map $j: X^{1} \rightarrow M \in \mathscr{V} / k$ such that:

(i) The restriction of $j$ to $P X^{1}$ induces $h: N P X^{1} \rightarrow M$. 
(ii) The composite $X^{0} \stackrel{d^{1}}{\rightarrow} X^{1} \stackrel{j}{\rightarrow} M$ is zero.

Let $j: X^{1} \rightarrow U(M)$ be the map of coalgebras induced by $j$, and define $g: X^{0} \rightarrow U(M)$ by $g=j d^{0}$.

We claim that

$$
k \longrightarrow X^{-1} \stackrel{d^{0}}{\longrightarrow} X^{0} \stackrel{g}{\longrightarrow} U(M) \longrightarrow k
$$

is an injective extension sequence, and this will show $C=X^{-1}$ is nice. The map

$$
c g: c X^{0} \rightarrow c U(M)
$$

of constant cosimplicial objects has mapping cone (3.7) $\boldsymbol{M}(\boldsymbol{c g})$. Letting $\tilde{\boldsymbol{X}}$ equal $\boldsymbol{X}$ without augmentation, there is a unique cosimplicial map

over $\mathscr{C} \mid k$ such that:

$$
F: \tilde{X} \rightarrow M(c g)
$$

(i) $F^{0}=1: X^{0} \rightarrow X^{0}$.

(ii) The composite

$$
X^{1} \stackrel{F^{1}}{\longrightarrow} X^{0} \otimes U(M) \stackrel{\alpha \otimes 1}{\longrightarrow} k \otimes U(M)
$$

equals $\bar{j}$ where $\alpha: X^{0} \rightarrow k$ is the counit.

It is easily shown that

$$
N P F^{*}: H^{n}(N P \tilde{X}) \rightarrow H^{n}(N P M(c g))
$$

is an isomorphism for $n \leqq 2$. Hence by 3.5

$$
F^{*}: H^{n}(\tilde{X}) \rightarrow H^{n}(M(c g))
$$

is an isomorphism for $n \leqq 1$. Since $H^{0}(\tilde{X}) \approx X^{-1}$ and $H^{1}(\tilde{X})=0$, it follows by 3.8 that

$$
X^{0} \square_{U(M)} k \approx X^{-1}, \quad \operatorname{Cotor}_{-1, *}^{U(M)}\left(X^{0}, k\right)=0,
$$

where $g: X^{0} \rightarrow U(M)$ gives the comodule structure. Hence 4.3 shows that $\left(^{*}\right)$ is an injective extension sequence.

Remark 4.5. Suppose $C \in \mathscr{C} \mid k$ is nice and $i: C \rightarrow B$ is any monomorphism in $\mathscr{C} / k$ with $B$ cofree. Then there is an injective extension sequence

$$
k \longrightarrow C \stackrel{i}{\longrightarrow} B \stackrel{j}{\longrightarrow} B^{\prime \prime} \longrightarrow k
$$

with $B^{\prime \prime}$ cofree. This may be shown by constructing a cosimplicial resolution $\boldsymbol{X}$ of $C$ with

$$
\left(\boldsymbol{X}^{-1} \stackrel{d^{0}}{\longrightarrow} \boldsymbol{X}^{0}\right)=(C \stackrel{i}{\longrightarrow} B)
$$

and then using $X$ in the proof of 4.4 .

5. A spectral sequence for Cotor. For a homology coalgebra $C \in \mathscr{C} / k$ we shall construct a spectral sequence of Hopf algebras $\left\{E^{r}(C)\right\}$ converging to $\operatorname{Cotor}^{c}(k, k)$ 
and such that $E^{2}(C)$ depends only on $R^{*} P(C)$. This technical machinery will be applied in $\$ 6$.

5.1. Recollections on Cotor. For $C \in \mathscr{C} \mid k$ there is defined (see [7], [12]) a second quadrant bigraded Hopf algebra

$$
\operatorname{Cotor}^{C}(k, k)=\left\{\operatorname{Cotor}_{n, q}^{C}(k, k)\right\}_{n \leqq 0 \leqq q}
$$

where $n$ is the homological degree, and $n+q$ is the total degree. By [12]

$$
\operatorname{Cotor}^{C}(k, k)=H(\Omega C)
$$

where $\Omega(C)$ is the differential Hopf algebra given by the reduced cobar construction. Thus $\Omega(C)$ is a bigraded tensor algebra with

$$
\Omega_{-n, *}(C)=\bigotimes^{n}(J C)
$$

where the comultiplication is determined by the condition $\Omega_{-1, *}(C) \subset P \Omega(C)$, and where the differential is of degree $(-1,0)$.

If $C$ is cofree, it is well known (see [12]) that

$$
\operatorname{Cotor}^{C}(k, k) \approx G(P C)
$$

where $G$ is the following functor from $\mathscr{V} / k$ to bigraded Hopf algebras. If $k$ is of characteristic 2 , then $G(M)$ is the exterior algebra generated by $G_{-1, *}(M)=M$; and if $k$ is of characteristic not 2, then $G(M)$ is the free commutative algebra generated by $G_{-1, *}(M)=M$. The comultiplication of $G(M)$ is determined by the condition that $G_{-1, *}(M) \subset P G(M)$.

5.2. The Hopf algebra $E^{2}(C)$. For $C \in \mathscr{C} \mid k$ we now construct the $E^{2}$-term of our spectral sequence (5.3). Consider the cosimplicial object $G P \widetilde{K} C$ over the category of bigraded Hopf algebras, with $\tilde{K} C$ as in 2.4. Define

$$
E_{s, n, q}^{2}(C)=H^{-s}\left(G_{n, q} P \widetilde{K} C\right), \quad s, n \leqq 0 \leqq q,
$$

with $s+n+q$ called the total degree, and give $E^{2}(C)$ the following structure as a trigraded Hopf algebra. Let $\varphi^{\prime}$ denote the composition

$$
\begin{gathered}
H^{-s}\left(G_{n, q} P \tilde{K} C\right) \otimes H^{-h}\left(G_{i, j} P \tilde{K} C\right) \stackrel{f}{\longrightarrow} H^{-s-h}\left(G_{n, q} P \tilde{K} C \otimes G_{i, j} P \widetilde{K} C\right) \\
\stackrel{\mu^{*}}{\longrightarrow} H^{-s-h}\left(G_{n+i, q+j} P \widetilde{K} C\right)
\end{gathered}
$$

where $f$ is induced by the dual to the Alexander-Whitney map [8, p. 241] and $\mu$ is the multiplication in $G(\cdot)$. The multiplication $\varphi$ of $E^{2}(C)$ is defined by

$$
\varphi=(-1)^{s(i+j)} \varphi^{\prime}: E_{s, n, q}^{2}(C) \otimes E_{h, i, j}^{2}(C) \rightarrow E_{s+h, n+i, q+j}^{2}(C) .
$$

Let $\Delta^{\prime}$ denote the composition

$$
\begin{aligned}
H^{-s-h}\left(G_{n+i, q+j} P \widetilde{K} C\right) & \stackrel{\psi^{*}}{\longrightarrow} H^{-s-h}\left(G_{n, q} P \tilde{K} C \otimes G_{i, j} P \tilde{K} C\right) \\
& \stackrel{g}{\longrightarrow} H^{-s}\left(G_{n, q} P \tilde{K} C\right) \otimes H^{-h}\left(G_{i, j} P \tilde{K} C\right)
\end{aligned}
$$


where $\psi$ is a component of the diagonal map of $G(\cdot)$, and $g$ is induced by the dual to the shuffle map [8, p. 243]. The diagonal map $\Delta$ of $E^{2}(C)$ has components defined by

$$
\Delta=(-1)^{s(i+j)} \Delta^{\prime}: E_{s+h, n+i, q+j}^{2}(C) \rightarrow E_{s, n, q}^{2}(C) \otimes E_{h, i, j}^{2}(C) .
$$

One shows that $E^{2}(C)$ is a Hopf algebra with commutative multiplication and commutative diagonal. (The twisting map is defined using total degrees.) Clearly

$$
\begin{aligned}
E_{s, 0, q}^{2}(C) & =k \quad \text { if } s, q=0, \\
& =0 \quad \text { otherwise; } \\
E_{-s,-1, *}^{2}(C) & =R^{s} P(C) .
\end{aligned}
$$

In general $E^{2}(C)$ depends only on $R^{*} P(C)$ since by [5] $H^{*}(G P \widetilde{K} C)$ depends only on $H^{*}(P \tilde{K} C)$.

THeOREM 5.3. For $C \in \mathscr{C} \mid k$ there is a natural spectral sequence $\left\{E^{r}(C), d^{r}\right\}_{r \geqq 2}$ of trigraded differential Hopf algebras such that:

(i) $E^{2}(C)$ is given by 5.2 .

(ii) The differential $d^{r}$ is of degree $(-r, r-1,0)$.

(iii) The spectral sequence $\left\{E^{r}(C)\right\}$ converges to $\operatorname{Cotor}^{C}(k, k)$ in the naive sense, i.e. there is a decreasing filtration on $\operatorname{Cotor}^{C}(k, k)$ compatible with the Hopf algebra structure and with

$$
E^{0} \operatorname{Cotor}^{C}(k, k) \approx E^{\infty}(C)
$$

as Hopf algebras. In particular $E_{s, n, q}^{\infty}(C)$ is a subquotient of $\operatorname{Cotor}_{s+n, q}^{C}(k, k)$.

(iv) The image of the edge morphism

$$
R^{s} P(C)=E_{-s,-1, *}^{2}(C) \rightarrow \operatorname{Cotor}_{-s-1, *}^{C}(k, k)
$$

lies in $P$ Cotor $^{C}(k, k)$.

Proof. Let $\boldsymbol{Y}$ denote the cosimplicial object $\tilde{K} C$. Let $T(\boldsymbol{Y})$ be the trigraded $k$-module

$$
T_{s, n, q}(\boldsymbol{Y})=\Omega_{n, q}\left(\boldsymbol{Y}^{-s}\right), \quad s, n \leqq 0 \leqq q
$$

with $\Omega(\cdot)$ as in 5.1 , and with $s+n+q$ called the total degree. The total differential $\partial_{T}$ on $T_{s, n, q}(\boldsymbol{Y})$ is given by

$$
\partial_{T}=\partial+(-1)^{n+q} \delta
$$

where $\partial: T_{s, n, q}(\boldsymbol{Y}) \rightarrow T_{s, n-1, q}(\boldsymbol{Y})$ is the differential of $\Omega\left(\boldsymbol{Y}^{-s}\right)$ and

$$
\delta: T_{s, n, q}(Y) \rightarrow T_{s-1, n, q}(Y)
$$

is the coboundary for the cosimplicial object $\Omega_{n, q}(\boldsymbol{Y})$. Let $\varphi^{\prime}$ denote the composition

$$
\Omega_{n, q}\left(\boldsymbol{Y}^{-s}\right) \otimes \Omega_{i, j}\left(\boldsymbol{Y}^{-h}\right) \stackrel{f}{\longrightarrow} \Omega_{n, q}\left(\boldsymbol{Y}^{-s-h}\right) \otimes \Omega_{i, j}\left(\boldsymbol{Y}^{-s-h}\right) \stackrel{\mu}{\longrightarrow} \Omega_{n+i, q+j}\left(\boldsymbol{Y}^{-s-h}\right)
$$

where $f$ is dual to the Alexander-Whitney map [8, p. 241] and $\mu$ is the multiplication in $\Omega(\cdot)$. The multiplication map

$$
\varphi=(-1)^{s(i+j)} \varphi^{\prime}: T_{s, n, q}(\boldsymbol{Y}) \otimes T_{h, i, j}(\boldsymbol{Y}) \rightarrow T_{s+h, n+i, q+j}(\boldsymbol{Y})
$$


makes $\left(T(\boldsymbol{Y}), \partial_{T}\right)$ a differential graded algebra. Similarly $\left(T(\boldsymbol{Y}), \partial_{T}\right)$ is a differential graded coalgebra with diagonal map defined using the dual to the shuffle map [8, p. 243] and the diagonal in $\Omega(\cdot)$. We do not claim that $T(Y)$ is a Hopf algebra.

Filter $T(Y)$ by $\left\{F_{s} T Y\right\}_{s \leqq 0}$ where $F_{s} T Y$ is generated as a graded $k$-module by all $T_{m, n, q}(Y)$ with $m \leqq s$. The filtration is compatible with the algebra and coalgebra structures of $T(Y)$ and we claim that the associated spectral sequence satisfies 5.3. By construction, each $E^{r}(C)$ is a differential algebra and coalgebra; and one easily shows $E^{2}(C)$ is the Hopf algebra of 5.2. Hence $E^{r}(C), r \geqq 2$, is a Hopf algebra. The convergence condition 5.3 (iii) follows from the fact that $T(Y)$ has total homology satisfying

$$
H(T Y) \approx \operatorname{Cotor}^{C}(k, k)
$$

which may be proved by a spectral sequence argument. Part 5.3 (iv) follows since elements of $E_{s,-1, q}^{\infty}(C)$ are represented by cycles in $T_{s,-1, q}(Y)$ which must be primitive in $T(Y)$ for reasons of degree.

6. Cotor for nice coalgebras. For a homology $k$-coalgebra $C$, it is well known (see [12]) that the Hopf algebra $\operatorname{Cotor}^{C}(k, k)$ is primitively generated. Let

$$
P_{*, *} C=P \operatorname{Cotor}^{C}(k, k)
$$

be the bigraded Lie algebra of primitive elements, and note that $P_{*, *} C$ is restricted [11] if the characteristic of $k$ is $p \neq 0$. By [11] the Hopf algebra $\operatorname{Cotor}^{C}(k, k)$ is completely determined by the (restricted) Lie algebra $P_{*, *} C$.

THEOREM 6.1. If $C \in \mathscr{C} \mid k$ with $k$ of characteristic 0 , there are natural isomorphisms $R^{s} P(C) \approx P_{-s-1, *} C, s \geqq 0$.

REMARK. If $C$ is nice in 6.1 , then the computation of $\operatorname{Cotor}^{C}(k, k)$ is reduced to determining $P_{-1, *} C=P C, P_{-2, *} C$, and the Lie product

$$
[,]: P_{-1, *} C \otimes P_{-1, *} C \rightarrow P_{-2, *} C .
$$

Proof. In the spectral sequence 5.3,

$$
E_{-s,-1, *}^{2}(C) \approx R^{s} P(C), \quad s \geqq 0,
$$

and it is straightforward to show using [5] that $E^{2}(C)$ is the free commutative algebra generated by $E_{*,-1, *}^{2}(C)$. Thus $E^{2}(C)$ is the tensor product of a polynomial algebra generated by elements of even total degree in $E_{*,-1, *}^{2}(C)$ with an exterior algebra generated by elements of odd total degree. It follows that $E^{2}(C)=E^{\infty}(C)$ and so the edge morphism

$$
\sigma: R^{s} P(C) \rightarrow P_{-s-1, *} C, \quad s \geqq 0,
$$

is monomorphic. Using the structure of $E^{\infty}(C)$, together with the PoincaréBirkhoff-Witt theorem [11] applied to $\operatorname{Cotor}^{C}(k, k)$, one easily shows that $\sigma$ is an isomorphism. 
THEOREM 6.2. Let $C \in \mathscr{C} \mid k$ be nice with $k$ of characteristic 2. Then $P_{-1, *} C$ $\approx R^{0} P(C), P_{-2, *} C \approx R^{1} P(C)$, and $P_{-n, *} C=0$ unless $n=2^{t}$ with $t \geqq 0$. If $\left\{x_{i}\right\}_{i \in I}$ is any homogeneous $k$-basis for $P_{-2, *} C$, then $\left\{x_{i}^{\left[2^{t}\right]}\right\}_{i \in I}$ is a $k$-basis for $P_{-2^{t+1}, *} C$. Furthermore all Lie products in $P_{*, *} C$ are trivial except (possibly) those of the form

$$
[,]: P_{-1, *} C \otimes P_{-1, *} C \rightarrow P_{-2, *} C .
$$

Proof. In the spectral sequence 5.3,

$$
E_{-s,-1, *}^{2}(C) \approx R^{s} P(C), \quad s \geqq 0,
$$

and $R^{s} P(C)=0$ for $s \geqq 2$. It is straightforward to show using [5] that $E^{2}(C)$ is the tensor product of the exterior algebra generated by $E_{0,-1, *}^{2}(C)$ with the polynomial algebra generated by $E_{-1,-1, *}^{2}(C)$. Thus $E^{2}(C)=E^{\infty}(C)$ and 6.2 follows easily.

A coalgebra $C \in \mathscr{C} \mid k$ will be called regular [12] if $C$ is nice and $R^{1} P(C)$ is trivial in odd degrees.

For $C \in \mathscr{C} \mid k$ let $P_{*, *}^{+} C$ be the subobject of $P_{*, *} C$ consisting of all elements of even total degree.

THEOREM 6.3. Let $C \in \mathscr{C} \mid k$ be regular with $k$ of characteristic $p$ odd. Then $P_{-1, *} C$ $\approx R^{0} P(C), P_{-2, *} C \approx R^{1} P(C)$, and $P_{-n, *} C=0$ unless either $n=p^{t}$ or $n=2 p^{t}$ with $t \geqq 0$. If $\left\{x_{i}\right\}_{i \in I}$ is any homogeneous $k$-basis for $P_{-1, *}^{+} C$ (resp. $\left.P_{-2, *} C\right)$, then for $t \geqq 1$, $\left\{x_{i}^{\left[p^{t}\right]}\right\}_{i \in I}$ is a $k$-basis for $P_{-p^{t}, *} C$ (resp. $\left.P_{-2 p^{t}, *} C\right)$. Furthermore, all Lie products in $P_{*, *} C$ are trivial except (possibly) those of the form

$$
[,]: P_{-1, *} C \otimes P_{-1, *} C \rightarrow P_{-2, *} C .
$$

Proof. In the spectral sequence 5.3, it is straightforward to show by [5] that $E^{2}(C)$ is the free commutative algebra generated by $E_{0,-1, *}^{2}(C)=R^{0} P(C)$ and by $E_{-1,-1, *}^{2}(C) \approx R^{1} P(C)$. Thus $E^{2}(C)=E^{\infty}(C)$ and 6.3 follows easily.

REMARK 6.4. Under the hypotheses of 6.2 and 6.3, the problem of computing Cotor $^{C}(k, k)$ is reduced to that of determining $P_{-1, *} C=P C, P_{-2, *} C$,

$$
[,]: P_{-1, *} C \otimes P_{-1, *} C \rightarrow P_{-2, *} C
$$

and in 6.2()$^{[2]}: P_{-1, *} C \rightarrow P_{-2, *} C$.

\section{APPENDIX}

7. Derived functors of nonadditive functors. Let

$$
T: \mathscr{C} \rightarrow \mathscr{A}
$$

be a (covariant) functor from an arbitrary category $\mathscr{C}$ to an abelian category $\mathscr{A}$. If $\mathscr{C}$ is equipped with a class $\mathscr{M}$ of injective models (7.2) we will define right derived functors of $T$ with respect to $\mathscr{M}$

$$
R^{n} T: \mathscr{C} \rightarrow \mathscr{A}, \quad n \geqq 0 .
$$

Let $\mathscr{C}^{+}$denote the category whose objects are the same as those of $\mathscr{C}$ and whose maps are formal sums of maps in $\mathscr{C}$, i.e. $\operatorname{Hom}_{\mathscr{C}^{+}}(X, Y)$ is the free abelian 
group on $\operatorname{Hom}_{\mathscr{C}}(X, Y)$. Thus $\mathscr{C}$ is a subcategory of $\mathscr{C}^{+}$, and $T$ extends to a unique additive functor

$$
T^{+}: \mathscr{C}^{+} \rightarrow \mathscr{A}
$$

Definition 7.1. If $\mathscr{M}$ is a class of objects in $\mathscr{C}$, a right $\mathscr{M}$-resolution for $N \in \mathscr{C}$ is a chain complex

$$
N \stackrel{\varepsilon}{\longrightarrow} M^{0} \stackrel{\delta}{\longrightarrow} M^{1} \stackrel{\delta}{\longrightarrow} \ldots
$$

in $\mathscr{C}^{+}$such that:

(i) For $n \geqq 0, M^{n} \in \mathscr{M}$.

(ii) For each $M \in \mathscr{M}$ the functor $\operatorname{Hom}_{\mathscr{C}^{+}}(\cdot, M)$ transforms $\left(^{*}\right)$ into an acyclic complex of abelian groups

$$
0 \leftarrow \operatorname{Hom}_{\mathscr{C}^{+}}(N, M) \leftarrow \operatorname{Hom}_{\mathscr{C}}+\left(M^{0}, M\right) \leftarrow \operatorname{Hom}_{\mathscr{C}}+\left(M^{1}, M\right) \leftarrow \cdots .
$$

Definition 7.2. A class of injective models for $\mathscr{C}$ consists of a class $\mathscr{M}$ of objects in $\mathscr{C}$ such that each $N \in \mathscr{C}$ has a right $\mathscr{M}$-resolution.

7.3. Right derived functors. Let

$$
T: \mathscr{C} \rightarrow \mathscr{A}
$$

be as above, and let $\mathscr{M}$ be a class of injective models for $\mathscr{C}$. The right derived functors of $T$ with respect to $\mathscr{M}$ are defined by the usual Cartan-Eilenberg [4] procedure. In particular for $N \in \mathscr{C}, R^{n} T(N)$ is isomorphic to the $n$th cohomology group of

$$
0 \longrightarrow T^{+}\left(M^{0}\right) \stackrel{T^{+}(\delta)}{\longrightarrow} T^{+}\left(M^{1}\right) \stackrel{T^{+}(\delta)}{\longrightarrow} \ldots
$$

where

$$
N \stackrel{\varepsilon}{\longrightarrow} M^{0} \stackrel{\delta}{\longrightarrow} M^{1} \stackrel{\delta}{\longrightarrow} \cdots
$$

is a right $\mathscr{M}$-resolution of $N$. The obvious comparison theorem holds for right $\mathscr{M}$-resolutions and we obtain functors

$$
R^{n} T: \mathscr{C} \rightarrow \mathscr{A}, \quad n \geqq 0,
$$

unique up to natural equivalence. Furthermore $\varepsilon: N \rightarrow M^{0}$ induces a natural map $\varepsilon: T(N) \rightarrow R^{0} T(N)$.

It is easy to show:

Proposition 7.4. For $M \in \mathscr{M}, \varepsilon: T(M) \rightarrow R^{0} T(M)$ is isomorphic and $R^{n} T(M)=0$ for $n>0$.

REMARK 7.5. If $\mathscr{C}$ is an abelian category with enough injectives, then [1] the injectives form a class of injective models for $\mathscr{C}$, and the above right derived functors coincide with the classical [4] ones when $T$ is additive.

7.6. Cosimplicial objects. In studying derived functors it is convenient to use cosimplicial methods. A cosimplicial object $\boldsymbol{X}$ (over a category $\mathscr{C}$ ) consists of 
(i) for every integer $n \geqq 0$ an object $\boldsymbol{X}^{n} \in \mathscr{C}$.

(ii) for every pair of integers $(i, n)$ with $0 \leqq i \leqq n$ coface and codegeneracy maps

$$
d^{i}: X^{n-1} \rightarrow X^{n} \in \mathscr{C}, \quad s^{i}: X^{n+1} \rightarrow X^{n} \in \mathscr{C},
$$

satisfying the identities

$$
\begin{aligned}
d^{j} d^{i} & =d^{i} d^{j-1}, & & i<j, \\
s^{j} d^{i} & =d^{i} s^{j-1}, & & i<j, \\
& =i d, & & i=j, j+1, \\
& =d^{i-1} s^{j}, & & i>j+1, \\
s^{j} s^{i} & =s^{i-1} s^{j}, & & i>j .
\end{aligned}
$$

Thus a cosimplicial object over $\mathscr{C}$ corresponds to a simplicial (see [10]) object over the dual category $\mathscr{C}^{*}$.

An augmentation for $\boldsymbol{X}$ consists of a map $d^{0}: \boldsymbol{X}^{-1} \rightarrow \boldsymbol{X}^{0} \in \mathscr{C}$ such that

$$
d^{1} d^{0}=d^{0} d^{0}: X^{-1} \rightarrow X^{1} .
$$

If $\boldsymbol{X}$ is an (augmented) cosimplicial object over $\mathscr{C}$, there is a chain complex $c h^{+} \boldsymbol{X}$ over $\mathscr{C}^{+}$defined by

$$
c h^{+} X^{n}=X^{n}, \quad \delta=\sum_{i=0}^{n+1}(-1)^{i} d^{i}: c h^{+} X^{n} \rightarrow c h^{+} X^{n+1}
$$

for $n \geqq 0$ (for $n \geqq-1$ if $\boldsymbol{X}$ augmented).

If $\mathscr{C}$ is an abelian category there is a chain complex $\operatorname{ch} X$ over $\mathscr{C}$ defined by omitting the " + " in the definition of $c h^{+} X$; and we then let $H^{n}(X)$ denote $H^{n}(\operatorname{ch} X)$.

7.7. Triples and cosimplicial objects. For arbitrary categories $\mathscr{C}$ and $\mathscr{D}$ let

$$
F: \mathscr{C} \rightarrow \mathscr{D}, \quad G: \mathscr{D} \rightarrow \mathscr{C},
$$

be functors with $F$ left adjoint to $G$, and denote the adjunction maps by

$$
\varphi: 1_{\mathscr{C}} \rightarrow G F, \quad \psi: F G \rightarrow 1_{\mathscr{D}} .
$$

There is associated a triple [6]

where

$$
K: \mathscr{C} \rightarrow \mathscr{C}, \quad \varphi: 1_{\mathscr{C}} \rightarrow K, \quad \mu: K K \rightarrow K,
$$

$$
K=G F, \quad \mu=1 \psi 1: G(F G) F \rightarrow G 1 F .
$$

For $N \in \mathscr{C}$ let $K^{0} N=N$ and $K^{n} N=K K^{n-1} N$. Define an augmented cosimplicial object $\boldsymbol{K} N$ by:

$$
\begin{aligned}
& K N^{n}=K^{n+1} N, \quad n \geqq-1, \\
& \left(K N^{n-1} \longrightarrow d^{i} \longrightarrow K N^{n}\right)=\left(K^{n} N \stackrel{K^{i} \varphi K^{n-i}}{\longrightarrow} K^{n+1} N\right), \quad 0 \leqq i \leqq n, \\
& \left(K N^{n+1} \stackrel{s^{i}}{\longrightarrow} K N^{n}\right)=\left(K^{n+2} N \stackrel{K^{i} \mu K^{n-i}}{\longrightarrow} K^{n+1} N\right), \quad 0 \leqq i \leqq n .
\end{aligned}
$$


The following proposition provides many examples in which the above theory of derived functors may be applied. We assume the conditions of 7.7.

Proposition 7.8. Let $\mathscr{M}$ be the class of all $M \in \mathscr{C}$ with $M \approx G D$ for some $D \in \mathscr{D}$. Then $\mathscr{M}$ is a class of injective models for $\mathscr{C}$, and $\mathrm{ch}^{+}(\boldsymbol{K} N)$ is a right $\mathscr{M}$-resolution for $N \in \mathscr{C}$.

Proof. Clearly $K N^{n} \in \mathscr{M}$ for all $n \geqq 0$, so it suffices to show that

$$
\operatorname{Hom}_{\mathscr{C}^{+}}\left(c h^{+}(\boldsymbol{K} N), G D\right)
$$

is an acyclic chain complex for each $D \in \mathscr{D}$. A contracting homotopy

$$
\bar{s}: \operatorname{Hom}_{\mathscr{C}^{+}}\left(\operatorname{ch}^{+}(K N)^{n-1}, G D\right) \rightarrow \operatorname{Hom}_{\mathscr{C}^{+}}\left(\operatorname{ch}^{+}(K N)^{n}, G D\right)
$$

for $n \geqq 0$ is induced by the following map

$$
s: \operatorname{Hom}_{\mathscr{C}}\left(K^{n} N, G D\right) \rightarrow \operatorname{Hom}_{\mathscr{C}}\left(K^{n+1} N, G D\right) .
$$

For $f: K^{n} N \rightarrow G D \in \mathscr{C}$ let $s(f)=G\left(f^{\prime}\right): G F K^{n} N \rightarrow G D$ where $f^{\prime}$ is adjoint to $f$.

REMARK 7.9. Under the hypotheses of 7.8, right derived functors with respect to $\mathscr{M}$

$$
R^{n} T: \mathscr{C} \rightarrow \mathscr{A}, \quad n \geqq 0,
$$

may be constructed as follows. For $N \in \mathscr{C}$

$$
R^{n} T(N) \approx H^{n}(T \tilde{K} N)
$$

where $T \widetilde{K} N$ is the cosimplicial object over $\mathscr{A}$ obtained from $K N$ by omitting the augmentation and applying $T$ dimensionwise.

\section{REFERENCES}

1. M. André, Méthode simpliciale en algèbre homologique et algèbre commutative, Lecture Notes in Math., no. 32, Springer-Verlag, Berlin, 1967. MR 35 \#5493.

2. A. Bousfield and E. Curtis, A spectral sequence for the homotopy of nice spaces, Trans. Amer. Math. Soc. (to appear).

3. A. Bousfield and D. Kan, The homotopy spectral sequence of a space with coefficients in a ring, (to appear).

4. H. Cartan and S. Eilenberg, Homological algebra, Princeton Univ. Press, Princeton. N. J., 1956. MR 17, 1040.

5. A. Dold and D. Puppe, Homologie nicht-additiver Funktoren. Anwendungen, Ann. Inst. Fourier Grenoble 11 (1961), 201-312. MR 27 \#186.

6. S. Eilenberg and J. C. Moore, Adjoint functors and triples, Illinois J. Math. 9 (1965), 381-398. MR 32 \#2455.

7. - Homology and fibrations. I: Coalgebras, cotensor product and its derived functors, Comment. Math. Helv. 40 (1966), 199-236. MR 34 \#3579.

8. S. MacLane, Homology, Die Grundlehren der math. Wissenschaften, Band 114, Academic Press, New York and Springer-Verlag, Berlin, 1963. MR 28 \#122.

9. W. Massey and F. Peterson, The mod 2 cohomology structure of certain fibre spaces, Mem. Amer. Math. Soc. No. 74 (1967). MR 37 \#2226. 
10. J. P. May, Simplicial objects in algebraic topology, Van Nostrand Math. Studies, no. 11, Van Nostrand, Princeton, N. J., 1967. MR 30 \#5942.

11. J. Milnor and J. C. Moore, On the structure of Hopf algebras, Ann. of Math. (2) 81 (1965), 211-264. MR 36 \#4259.

12. J. C. Moore and L. Smith, Hopf algebras and multiplicative fibrations. II, Amer. J. Math. 90 (1968), 1113-1150.

13. D. Quillen, Homotopical algebra, Lecture Notes in Math., no. 43, Springer-Verlag, Berlin, 1967. MR 36 \#6480.

14. D. Rector, An unstable Adams spectral sequence, Topology 5 (1966), 343-346. MR 33 \#8003.

15. L. Smith, Homological algebra and the Eilenberg-Moore spectral sequence, Trans. Amer. Math. Soc. 129 (1967), 58-93. MR 35 \#7337.

16. D. Quillen, On the (co-)homology of commutative rings, Proc. Sympos. Pure Math., vol. 17, Amer. Math. Soc., Providence, R. I., 1969.

BRANDEIS UNIVERSITY,

Waltham, Massachusetts 02154 